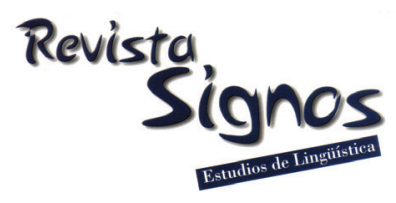

\title{
Teorías implícitas acerca de la comprensión de textos: Estudio exploratorio en estudiantes universitarios de primer año ${ }^{1}$
}

\author{
Implicit theories about reading comprehension: \\ Exploratory study in university students
}

\author{
Margarita Makuc Sierralta \\ Universidad de Magallanes \\ CHILE \\ margarita.makuc@umag.cl
}

\author{
Elisa Larrañaga Rubio \\ Universidad Castilla-La Mancha \\ ESPAÑA \\ elisa.larranaga@uclm.es
}

Recibido: 13-V-2013 / Aceptado: 26-V-2014

\section{Resumen}

En este artículo se presentan los resultados de la primera etapa del estudio realizado para elaborar un perfil lector de estudiantes universitarios. Inicialmente, se plantea determinar las teorías implícitas sobre comprensión de textos que predominan en estudiantes al ingreso a la universidad. Nos parece relevante establecer las teorías predominantes según ámbito del conocimiento y estudiar de qué modo el estudiante activa determinadas teorías según los tipos de discurso a los que se enfrenta en el contexto académico. A partir del supuesto de que la explicitación del conocimiento es un proceso gradual a través de la redescripción representacional que incluye tres niveles (reconocimiento, declarativo y procedimental) (Karmiloff-Smith, 1997), este estudio se focaliza en el nivel declarativo el cual se elicita mediante el Cuestionario de Teorías Implícitas. Los resultados del estudio permiten observar el predominio de la Teoría Lineal en los cuatro grupos de estudiantes. Ello evidencia que los estudiantes han configurado en su experiencia y formación una concepción de la lectura orientada a la reproducción fiel de contenidos, donde el rol del lector consiste en localizar información y transferirla a la memoria, privilegiando al texto como portador principal de significados. Por otra parte, se presentan diferencias que permiten relacionar las teorías implícitas con determinadas comunidades discursivas, aspecto que podría formar parte de la identidad de los diversos grupos de formación.

Palabras Clave: Teorías implícitas, comprensión textual, Teoría Lineal, Teoría Interactiva, Teoría Literaria. 


\begin{abstract}
This article presents the results of the first stage of a study intended to develop a reading profile of university students. We propose to recognize implicit theories based on text comprehension that predominate when students enter the university. It seems relevant to us to establish the prevailing theories text to the area of knowledge and how the student activates certain theories as stated by the types students face in the academic context. Based on the assumption that the explicitation of knowledge is a gradual process through representational redescription, which includes three levels (recognition, declarative and procedural) (Karmiloff-Smith, 1997). This study focuses on the declarative level which is elicited by means of the Implicit Theory Questionnaire. The results of the study allow to observe the predominance of the Linear Theory in the four groups students demonstrates that students have constructed in their experience a conception of reading focused on the faithful reproduction of contents, where the role of the reader consists of locating information and transferring it to the memory favouring the text as the main source of meaning. On the other hand, differences that allow for the connection between the implicit theories and specific discursive communities are also presented.
\end{abstract}

Key Words: Implicit theories, textual understanding Linear Theory, Interactive Theory, Literary Theory.

\title{
INTRODUCCIÓN
}

El presente estudio aborda la comprensión de textos escritos incorporando en su análisis recientes enfoques conceptuales focalizados en las 'teorías implícitas' que sostienen los sujetos sobre diversos dominios de la vida cotidiana (Rodrigo, 1985, 1997; Pozo, Pérez, Sanz \& Limón, 1992; Rodrigo, Rodríguez \& Marrero, 1993; Rodrigo \& Correa, 2001; Estrada, Yzerbit \& Serón, 2004; Castro, 2006; Marrero, 2009). Esta aproximación se realiza integrando diversos estudios sobre el conocimiento de los textos y los procesos cognitivos del lector que interactúan en la comprensión de textos (van Dijk, 2001; Kintsch, 2002, 2008; Parodi, 2007, 2009).

La comprensión de textos ha sido abordada desde diversas perspectivas teóricas (De Vega, 2004; Jamet, 2006; Marinkovic, Peronard \& Parodi, 2006; Hernández, 2008; Ibáñez, 2008; Eyzaguirre \& Fontaine, 2008; Velásquez, Cornejo \& Roco, 2008). La presente investigación aborda el estudio de la comprensión de textos desde la perspectiva del lector focalizando el análisis en las teorías implícitas de los lectores (Schraw \& Bruning, 1996; Schraw, 2000; Hernández, 2008; Makuc, 2008, 2011, 2012; Moore \& Narciso, 2011).

En este artículo interesa destacar el hecho de que los niveles de comprensión lectora y las estrategias consideradas eficaces se logran a partir de una permanente (re) elaboración de la experiencia por parte de los sujetos lectores, dando con ello origen a ‘teorías implícitas sobre la comprensión lectora', las que, a su vez, reflejarían la influencia 
de los modelos culturales predominantes en un determinado contexto social y campo disciplinario (Dubois, 1987; Wineburg, 1991; Carlino, 2003; Vélez, 2006; Hernández, 2008). El estudio de las teorías implícitas respecto de la comprensión textual permite, además, reconocer en los alumnos que inician los estudios universitarios aquellas teorías que en el ámbito de la formación escolar de enseñanza primaria y secundaria se han afianzado en los estudiantes como modelos culturales predominantes, influyendo tanto en las concepciones que los aprendices sostienen respecto de este dominio del conocimiento, como en las estrategias lectoras de los estudiantes.

Considerando lo anteriormente expuesto, el objetivo de este estudio se orienta a determinar las teorías implícitas sobre comprensión de textos que predominan en estudiantes universitarios al ingreso a la universidad. Asimismo, interesa abordar las teorías que predominan en los diversos grupos de formación, de manera de abordar los ámbitos del conocimiento y las teorías implícitas predominantes en las diversas disciplinas propias del contexto académico universitario.

\section{Las teorías implícitas: Diversos enfoques y conceptualizaciones}

En términos generales, la importancia de las 'teorías implícitas' radicaría en el hecho de que son un tipo de entidad cognitiva, inconsciente, latente e implícita, que ejercen una enorme influencia en las conductas, decisiones y acciones o respuestas de los 'sujetos' a determinados problemas del conocimiento (Karmiloff-Smith, 1997; Pozo, 2001; Rodrigo \& Correa, 2001; Castro, 2006; Martínez, 2009). El término 'teoría', en el sentido de las ideas que tienen las personas sobre las cosas y los sucesos cotidianos, ha sido abundantemente empleado en la literatura psicológica en los últimos años. Una de las pioneras en tratar de definir estas teorías en el dominio de los objetos y propiedades físicas, fueron Karmiloff-Smith y Inhelder (1984). Estas autoras destacan que las teorías implícitas ejercen una enorme influencia en la conducta (tanto en la reflexión como en la actuación) y en las respuestas a determinados problemas.

En el contexto europeo, se puede considerar a Groeben (1990) como uno de los precursores en esta línea de investigación. Este autor propone el concepto de 'teoría subjetiva', y la define desde una perspectiva cognitiva señalando que estas constituyen:

"cognitions relating to the self and the world constituting a complex aggregate with an (at least implicit) argumentational structure; these cognitions fulfil functions parallel to those of objetive "scientific" theories, namely those of explanation, prediction and technology" (Groeben, 1990: 21).

Reconociendo las diferencias entre los diversos constructos que describen este tipo de entidades cognitivas se destaca en los diversos enfoques el hecho de que compartirían el ser 'subjetivas' (Dann, 1990) por cuanto se basarían en el supuesto de que los sujetos al describir las razones y posibles consecuencias de sus acciones reconstruyen una teoría subjetiva. 
Al respecto, Catalán (2010) distingue las teorías subjetivas de las teorías implícitas, señalando que las teorías subjetivas describen el carácter posesivo y la procedencia de dichas teorías, mientras que las teorías implícitas describen un atributo relevante de las teorías que elabora un sujeto, esto es su carácter implícito predominante. No obstante lo anterior, tanto las teorías implícitas como las teorías subjetivas compartirían, diversas características: son relativamente estables, a menudo implícitas, modelan las acciones de las personas y se vinculan con la función social de fortalecimiento de la pertenencia al grupo (Dann, 1990; Rodríguez, 1993; Catalán, 2010).

El enfoque de 'teorías implícitas' en el marco de la psicología cognitiva propone que éstas estarían compuestas por conceptos y relaciones que, al igual las teorías científicas, tendrían como objetivo la interpretación y predicción del mundo circundante (Pozo, 2001, 2003). Recientemente, clarificando estas similitudes, Marrero (2009) señala que el sustrato epistemológico que guía el conocimiento cotidiano tiene una orientación eminentemente pragmática, mientras que en el conocimiento científico el foco es eminentemente teórico-conceptual (función pragmática v/s función epistémica). En otros términos, las teorías implícitas compartirían con el conocimiento científico o formal(izable), los marcos de garantía y justificación, pero se activarían en contextos propios de la vida cotidiana de los sujetos.

No obstante lo anterior, el hecho de que estas teorías no constituyan conocimiento racionalizado o elaborado conscientemente, no significa que conformen un conglomerado incidental de conocimiento carente de coherencia interna. Al contrario, el conocimiento cotidiano se diversificaría en cuanto a su contenido en un conjunto limitado de versiones, las que a su vez establecen una relación entre ambos tipos de conocimiento (racionalizado/cotidiano), evidenciando que estos no formarían compartimentos estancos, sino por el contrario, el mundo social facilitaría permanentes intercambios entre la ciencia y el sentido común. De este modo, las teorías implícitas se distinguen de las teorías científicas, en términos de su organización y carácter implícito, las que se entenderán como un modelo representacional que considera las representaciones del conocimiento cotidiano como un conjunto organizado y no como un conjunto de ideas aisladas (Pozo, Scheuer, Mateos \& PérezEcheverría, 2006; Scheuer \& Pozo, 2006). En consecuencia, la activación de estas representaciones en escenarios más o menos concretos posibilitaría variaciones en referencia a dominios, posiciones y contextos particulares, indicando un relativo 'pluralismo representacional' así como ajustes en función de las características del escenario. Diversos autores (Pozo \& Carretero, 1987; Marrero, 1988, 2009; Rodrigo et al., 1993; Rodrigo, 1997; Rodrigo \& Correa, 2001) proponen una serie de rasgos que distinguen una conceptualización de otra. Específicamente, para Rodrigo et al. (1993) las teorías implícitas serían un sistema de experiencias de dominio a partir de los cuales sintetizarían una determinada teoría para diferentes propósitos en determinados momentos. 
Dado el carácter situado de estas representaciones, las teorías implícitas no constituirían esquemas globales, sino redes de trazos que se integrarían en la memoria operativa con los trazos episódicos del 'modelo mental' de la situación, los que contendrían información sobre la estructura causal, temporal e intencional de la situación. Las teorías implícitas permitirán, por tanto, responder a las diversas y variables demandas cognitivas de la situación, producto de que la información prealmacenada y estereotipada es superada al integrarse en un modelo mental de la situación. La importancia de ello radicaría en que los 'modelos mentales' presentan la posibilidad de incrementarse y modificarse mediante una permanente negociación de significados, a medida que la situación varía, en un proceso de intercambio social, de las exigencias profesionales, del grado de experticia (Rodrigo, 1997; Rodríguez, 2001; Rodrigo \& Correa, 2001; Marrero, 2009).

Por otra parte, Pozo et al. (2006) plantean que las teorías implícitas constituirían más bien un nivel de representación previo, como un conjunto de principios generales e implícitos que se manifiestan con diversos grados de coherencia y consistencia según los contextos y situaciones. Estas concepciones se basarían en representaciones de naturaleza implícita. Al respecto, los autores sugieren destacar la distinción entre teorías implícitas y teorías de dominio: las teorías implícitas estarían regidas por principios epistemológicos, ontológicos y conceptuales que organizarían y restringirían la forma en que nos representamos las situaciones; las 'teorías de dominio' proporcionarían los rasgos invariantes de los modelos mentales y se organizarían y estructurarían a partir de una serie de supuestos o principios implícitos que constituirían la teoría implícita subyacente. De este modo, las teorías implícitas tendrían un carácter más general que las propias teorías de dominio, ya que las representaciones activadas por los sujetos en distintos dominios podrían compartir las mismas restricciones, en otras palabras distintas teorías de dominio pueden sustentarse en los mismos supuestos implícitos, así como los mismos principios pueden dar lugar a teorías de dominio con contenidos representacionales distintos.

La identificación de estos niveles se vincula con la naturaleza implícita de las representaciones, lo que permitiría comprender el cambio a nivel representacional mediante un proceso de explicitación progresiva de representaciones inicialmente implícitas, los autores sostienen que los niveles más cercanos a la acción, los más superficiales serían los más fáciles de explicitar, mientras que los niveles más profundos, esto es, los principios que articulan las teorías implícitas, serían los más difíciles de explicitar. Ello se explicaría según diversos autores (Rodrigo, 1985; Rodrigo et al., 1993) por el hecho de que estos constructos de naturaleza tácita son asequibles a la conciencia del sujeto solo bajo ciertas condiciones específicas (verbalización y reconocimiento, entre otras); de esta manera, los sujetos atribuirían valor de verdad a su teoría, sin reconocer su dimensión interpretativa.

La existencia de dos niveles funcionales, el de conocimiento y el de creencia es un aspecto fundamental del modelo representacional de las teorías implícitas (Triana \& 
Rodrigo, 1985; Marrero, 1988). Las síntesis de conocimientos representacionales (nivel representacional) constituye un repertorio de modelos culturales sobre un mismo fenómeno; así, por ejemplo, las diferentes teorías de los profesores sobre la enseñanza (Marrero, 1988, 2009) o las concepciones educativas de los padres (Triana \& Rodrigo, 1985; Triana, 1991) se organizan en torno a prototipos configurados por las ideas más típicas o representativas de una teoría. Las síntesis de creencia atribucionales (nivel atribucional) son versiones parciales de los modelos culturales conocidos sobre un mismo fenómeno, ya que en este nivel las personas asumen como propias algunas de las versiones que poseen a nivel representacional o bien una versión resumida de las mismas. Además, suelen permanecer implícitas, pues el individuo no tiene acceso consciente a la síntesis que ha hecho de su teoría (Marrero, 1988; Rodrigo, 1994a, 1994b).

La diferencia entre estos dos tipos de síntesis no radicaría en el contenido, ya que cualquier conocimiento podría ser sintetizado como representacional o atribucional, sino en el tipo de tarea cognitiva (demanda) que se requiera: teórica para las síntesis representacionales, pragmática para las atribucionales. Dadas sus características, las síntesis atribucionales resultarían especialmente difíciles de cambiar, ya que la ausencia de explicitación implica que el sujeto no ha reflexionado sobre la teoría que asume como propia, puesto que 'cree' en ella. Sin embargo, las síntesis de conocimiento, al no ser 'creencia', pueden ser sometidas a análisis, a crítica, y, por lo tanto, son susceptibles de cambio (Rodrigo et al., 1993). Estos dos niveles (representacional y atribucional) permitirían, entre otras cosas, analizar los procesos funcionales del cambio de conocimiento, pues la clave radicaría en la capacidad de acceder de modo consciente al contenido de las representaciones.

A partir del reconocimiento de estos niveles, Rodrigo (1993) sostiene que en las síntesis de conocimiento podemos identificar dos funciones principales: la comunicación interpersonal y el cambio de creencias. En cuanto a las síntesis de creencias se reconocen tres funciones: permiten la interpretación de sucesos, influyen de manera fundamental en los procesos cognitivos (comprensión, razonamiento, memoria); influyen en los fenómenos de representación de la acción, es decir, en la planificación de la acción y, en última instancia, en la acción misma.

En el contexto de los estudios realizados en docentes, se plantea que las teorías implícitas serían de naturaleza práctica y permitirían reducir la necesidad de procesamiento de información del profesor, al mismo tiempo intervendrían en su modo de planificar la enseñanza y en sus juicios y decisiones en la clase (García Jiménez, 1988; Mitchell, 1995).

Por último, el estudio de las teorías implícitas sobre la enseñanza y el aprendizaje, ha permitido observar que éstas son de naturaleza procedimental, situada y encarnada, en consecuencia muy difíciles de controlar conscientemente. Asimismo, su aprendizaje es implícito, pues serían el resultado de la experiencia personal y la 
educación informal en escenarios culturales de aprendizaje, en consecuencia, no suelen ser fáciles de comunicar ni de compartir, porque estarían representadas en códigos no formalizados (Pozo, 2001).

Dada la importancia de explicitar las teorías implícitas, diversos autores han destacado la propuesta de Karmiloff-Smith (1997) respecto de los diversos niveles de explicitación que permitirían dar cuenta de nuestros conocimientos acerca de un dominio determinado, a través de la teoría de la redescripción representacional. $\mathrm{Al}$ respecto, la autora sostiene que existiría predisposición innata específica correspondiente a ciertos microdominios que no pueden definirse como módulos encapsulados sino más bien como un 'sesgo o boceto en esqueleto' (Karmiloff-Smith, 1997) que, progresivamente, debido a factores externos e internos experimentarían un proceso de modularización haciéndose menos accesibles, más automáticos e independientes de otros sistemas. La idea central de la teoría de la redescripción representacional es que junto a este proceso de encapsulamiento se produce un proceso inverso de mayor apertura y accesibilidad, gracias a procesos mediante los cuales el sujeto va cambiando el formato de representación de la información que ha almacenado.

En otras palabras, el progreso de una teoría implícita a otra más avanzada requeriría reorganizar algunos de sus supuestos o principios básicos, promoviendo el cambio conceptual o representacional. Ya no se trataría -así- de adquirir conocimientos o de sustituir unos por otros más elaborados, sino -sobre todo- de aprender a activar esos conocimientos en los contextos adecuados (Rodrigo, 1994; Pozo \& Rodrigo, 2001; Jiménez, 2002; Pozo, 2003; Estrada et al., 2007; Contreras, 2010; Gómez \& Guerra, 2012).

\subsection{Teorías implícitas y comprensión textual: Un enfoque desde las concepciones del lector}

Diversos estudios (Schraw \& Bruning, 1996; Schraw, 2000; Vélez, 2006; Hernández, 2008; Makuc, 2008, 2011, 2012; Moore \& Narciso, 2011) han integrado las teorías acerca de la lectura como un referente para, posteriormente, analizar las teorías de los propios lectores. Estas investigaciones han logrado determinar que los lectores pueden agruparse en torno a diversas teorías que en mayor o menor medida se relacionan con la experiencia formativa del estudiante y con modelos culturales transmitidos durante el proceso de escolarización (Carlino, 2003; Peredo, 2003; Ruiz, 2009).

En este contexto, se destaca la investigación de Schraw y Bruning (1996) quienes plantean tres modelos epistémicos acerca de la lectura: de transmisión, translación y transaccional. El 'modelo de transmisión' se basa en la suposición de que el significado se transmite directamente del autor al lector, el lector se concibe como un receptor, cuyo objetivo principal es extraer el significado intencionado por el autor del texto más 
que construirlo activamente, una manifestación de este lector es que se focaliza en el significado literal. A diferencia del anterior, en el modelo de 'translación', el trabajo del lector es decodificar el mensaje presentado implícita o explícitamente en el texto sin hacer referencia a sus propias experiencias, el entorno cultural en que se redactó el texto o las intenciones del autor. Por último, el modelo 'transaccional' se basa en la suposición de que un texto adquiere diversos significados para diferentes lectores, no obstante las intenciones del autor o el contenido textual. Bajo este modelo, los lectores interpretan un texto desde la perspectiva de sus propias metas y objetivos dentro de un contexto particular, construyendo el significado con referencia al conocimiento previo del tema, experiencias lectoras anteriores y los objetivos de la situación actual.

En esta misma línea de estudios, Hernández (2008) identifica en los estudiantes de secundaria tres tipos de teorías: 'reproduccionista', 'interpretativa' y 'constructiva'. La teoría 'reproduccionista' conceptualiza la comprensión de textos como un acto de reproducción del significado/mensaje. La lectura consistiría en asistir al texto para instruirse de este y reproducir fielmente los contenidos que el autor expone. La principal función que se le reconoce a la comprensión lectora es 'transmitir saberes'. Por su parte, la teoría 'interpretativa' concibe la comprensión de textos como una actividad interpretativa que depende en gran medida del papel activo del lector en la búsqueda e interpretación del significado de los textos. El lector puede matizar de manera subjetiva lo que comprende, pero al final debe intentar reproducir lo que el texto dice. La función principal de la comprensión es aprender, su logro dependería, sobre todo, de la dimensión motivacional y cognitiva. La teoría 'constructiva', en cambio, plantea que la lectura constituye una actividad en que los significados son construidos por el lector gracias a lo que el autor dice en su texto; asimismo, el lector puede asumir actitudes valorativas o críticas sobre los textos o sobre aquello que dicen y cómo lo dicen los autores. La función principal de la comprensión es la comunicación. Se reconoce que leer es una herramienta de aprendizaje, para desarrollar instrumentos cognitivos y para ayudar a interpretar la realidad circundante.

Una similar clasificación de los modelos de lectura es abordada por Vélez (2006), quien identifica tres teorías en los relatos autobiográficos de estudiantes universitarios: la lectura como conjunto de habilidades, la lectura como un proceso interactivo y la lectura como proceso transaccional. La teoría que explica la lectura como 'conjunto de habilidades' concibe la lectura como un fenómeno observable en que el lector decodifica para extraer el significado que está en el texto y presenta un modelo secuencial y jerárquico de habilidades lectoras que se deben enseñar de manera gradual y acumulativa. Por su parte, el 'enfoque interactivo' propone un modelo sistémico de la lectura como procesamiento de la información. La información que ingresa debe ser procesada por los esquemas del sujeto. La lectura se considera un proceso activo, en que la mente procesa símbolos. Finalmente, el 'enfoque transaccional' concibe una representación cíclica de la lectura, en que no pueden separarse, lector, texto 
y contexto. Esta transacción adquiriría características de experiencia guiada por la postura que asume el lector dentro de un continuo entre la lectura estética y la lectura eferente.

En términos comparativos, las propuestas descritas (Schraw \& Bruning, 1996; Schraw, 2000; Vélez, 2006; Hernández, 2008) presentan similitudes en cuanto a que los diversos autores reconocen tres enfoques: un enfoque de transmisión (reproduccionista-conjunto de habilidades-lineal), un enfoque interpretativo (interactivo-de translación) y un tercer enfoque transaccional (constructivista). En nuestras investigaciones previas (Makuc, 2008, 2011, 2012, 2013), se han establecido tres teorías implícitas sobre la comprensión: Lineal, Interactiva y Literaria. Las dos primeras teorías coinciden en lo esencial con la clasificación y caracterización descrita por dichos estudio; sin embargo, la tercera teoría definida como Teoría Literaria correspondería a una dimensión que surge a partir de las afirmaciones de docentes y estudiantes que describen la lectura en términos de experiencias escolares, actividades didácticas, evaluativas, focalizadas en la lectura de textos literarios, promoviendo desarrollo de actitudes, valores, orientada al componente afectivo y actitudinal.

La Teoría Lineal y la Teoría Interactiva se caracterizan de acuerdo a referentes teórico-conceptuales en el ámbito de la comprensión de textos (Goodman \& Goodman, 1994; Pearson \& Stephens, 1994). Estas teorías presentan diferencias significativas, por cuanto representarían dos paradigmas en el enfoque de este constructo tanto en el nivel teórico como en la enseñanza (Pearson \& Stephens, 1994). La Teoría Lineal (Gough, 1994; Laberge \& Samuels, 1994; Linuesa \& Domínguez, 1999; Parodi, 1999) describe la primera etapa de los estudios sobre la comprensión textual. La lectura se concibe como un proceso perceptual directo, secuencial y ordenado. Esta Teoría se define como 'Lineal', pues los lectores se conciben como decodificadores de símbolos gráficos, los cuales deben ser traducidos a un código oral. En otras palabras, la comprensión del material escrito es, fundamentalmente, la comprensión del habla, producida en la mente del lector. Esta teoría, predominante en una primera etapa de los estudios sobre la comprensión, habría permitido plantear una concepción de lectura en la cual leer consistiría en transformar los signos gráficos en significados, enfatizando los procesos de reconocimiento de la palabra escrita.

Por otra parte, la Teoría Interactiva (van Dijk \& Kintsch, 1983; Goodman \& Goodman; 1994; Colomer \& Camps, 1996; Rumelhart, 1997; De Vega, 1998, 2004; Parodi, 2005, 2007) integra la flexibilidad característica de los sistemas perceptivos y cognitivos. Esta teoría supone que el procesamiento cognitivo del lector es de carácter descendente y ascendente, es decir, el procesamiento del texto escrito se realizaría a partir de los conocimientos que el lector activa durante la lectura y la información del texto. Ello supone un lector activo que procesa la información del texto basado en sus propios esquemas o marcos conceptuales emanados de su conocimiento y experiencia 
del mundo. En este enfoque la comprensión textual sería el resultado de mecanismos y procesos inferenciales, estrategias cognitivas, y lingüísticas (McKoon \& Ratcliff, 1998) que un lector experto activa de un modo estratégico e interactivo. Desde esta perspectiva, se identifican y describen las diversas dimensiones que interactúan en la comprensión de textos, elementos como la coherencia semántica y sintáctica; la macro y microestructura de un texto; la generación de inferencias y la caracterización de ellas (Paris, Lipson \& Wixson 1994; Colomer \& Camps, 1996; Parodi, 1999, 2007).

La Teoría Literaria se vincula con la lectura de textos literarios e implica, por tanto, capacidad imaginativa, identificación con personajes con situaciones de la historia y con el establecimiento de conexiones con la vida real. De esta forma, se plantea que comprender es imaginar, es crear imágenes. La comprensión sería posible solo si se presentan estados afectivo-actitudinales; comprender implica, además, comparar lecturas, personajes, identificar características asociadas a un personaje, describir la trama en la que están involucrados. Ello nos ubica en un tipo de lectura que tiende a promover un análisis literario, orientado a identificar personajes, acontecimientos, conflicto, desenlace, protagonista, antagonista, entre otras categorías.

Como podemos observar, la Teoría Lineal y la Teoría Interactiva coinciden con las clasificaciones desarrolladas por Schraw y Bruning (1996), Hernández (2008) y Vélez (2006). Estas teorías representarían dos concepciones opuestas en términos paradigmáticos y conceptuales, especialmente en cuanto al rol que atribuyen al lector. Al respecto, diversos autores (Winneburg, 1991; Pressley \& Afferbach, 1995; Bahloul, 2002) plantean que la Teoría Lineal presentaría un menor grado de plausibilidad respecto de desarrollar niveles superiores de comprensión lectora. La Teoría Interactiva, en cambio, constituiría un marco de referencia con mayores proyecciones en términos de promover niveles de lectura más profundos y con objetivos de aprendizaje.

A continuación, se presenta una síntesis de estas teorías estructurada en torno a tres conceptos centrales en la comprensión de textos: noción de comprensión, noción de lector y noción de texto. 
Tabla 1. Síntesis de teorías implícitas según la noción de comprensión, lector y texto

(Makuc, 2005, 2008).

\begin{tabular}{|c|c|c|c|}
\hline & Teoría Lineal & Teoría Interactiva & Teoría Literaria \\
\hline $\begin{array}{l}\text { Noción de } \\
\text { comprensión }\end{array}$ & $\begin{array}{l}\text { Leer consiste en transformar } \\
\text { signos gráficos en significados, } \\
\text { enfatizando los procesos de } \\
\text { reconocimiento de lo escrito. } \\
\text { La lectura se concibe como } \\
\text { proceso perceptual directo y } \\
\text { lineal, mediado por una trans- } \\
\text { formación, que produce un } \\
\text { código lingüístico y es tratado } \\
\text { por el cerebro como un proceso } \\
\text { de lenguaje. }\end{array}$ & $\begin{array}{l}\text { Desde esta teoría, no existen } \\
\text { razones para distinguir entre } \\
\text { la identificación de palabras y } \\
\text { la comprensión de frases, pues } \\
\text { lectura y comprensión son un } \\
\text { proceso complejo y no una tran- } \\
\text { scripción de signos gráficos. Esta } \\
\text { definición incorpora la dimen- } \\
\text { sión semántica y la búsqueda de } \\
\text { sentido que motiva a los lectores } \\
\text { a activar estrategias. }\end{array}$ & $\begin{array}{l}\text { Comprender es imaginar, disfrutar, } \\
\text { valorar estéticamente un texto. En } \\
\text { esta teoría, comprender implica } \\
\text { comparar lecturas, personajes, iden- } \\
\text { tificar tipos humanos, etc. La teoría } \\
\text { implícita literaria supone estrategias } \\
\text { orientadas a relacionar temas clási- } \\
\text { cos con temas actuales, estimular } \\
\text { la imaginación de los estudiantes } \\
\text { y promover la identificación con } \\
\text { problemáticas universales. }\end{array}$ \\
\hline $\begin{array}{l}\text { Noción de } \\
\text { lector }\end{array}$ & $\begin{array}{l}\text { Los lectores deben identificar } \\
\text { significados basándose en la in- } \\
\text { formación que el texto entrega. } \\
\text { Son, decodificadores de símbo- } \\
\text { los gráficos, los cuales deben ser } \\
\text { traducidos a un código oral. La } \\
\text { función del lector consiste en lo- } \\
\text { calizar información y transferirla } \\
\text { a la memoria. }\end{array}$ & $\begin{array}{l}\text { Los lectores intentan encontrar } \\
\text { el sentido a los textos. Por ello, } \\
\text { se definen por su capacidad para } \\
\text { hacer predicciones, superando el } \\
\text { procesamiento literal e interpre- } \\
\text { tando lo que leen a partir de sus } \\
\text { conocimientos previos. El lector } \\
\text { activo procesa la información } \\
\text { basado en esquemas emanados } \\
\text { de su experiencia. }\end{array}$ & $\begin{array}{l}\text { Dentro de este enfoque, un buen } \\
\text { lector se define como aquel que } \\
\text { es capaz de relacionar las situacio- } \\
\text { nes de una obra literaria con las } \\
\text { experiencias personales o relacio- } \\
\text { nar los autores clásicos con los de } \\
\text { actualidad: El lector disfruta con la } \\
\text { lectura, se compromete, es un lector } \\
\text { empático. }\end{array}$ \\
\hline $\begin{array}{l}\text { Noción de } \\
\text { texto }\end{array}$ & $\begin{array}{l}\text { El texto es considerado } \\
\text { portador de significados y } \\
\text { contiene toda la información } \\
\text { visual fonológica y semántica } \\
\text { necesaria para interpretarlo } \\
\text { correctamente. Para la identifi- } \\
\text { cación del significado que porta } \\
\text { el texto, el componente léxico es } \\
\text { fundamental. }\end{array}$ & $\begin{array}{l}\text { El texto se define como unidad } \\
\text { lingüística con sentido en virtud } \\
\text { de productores/ hablantes y } \\
\text { lectores/oyentes en contextos } \\
\text { particulares y con propósitos } \\
\text { definidos y con conocimientos } \\
\text { previos. Los textos son pro- } \\
\text { ducto de nuestra cognición y el } \\
\text { contexto. }\end{array}$ & $\begin{array}{l}\text { El texto debe ser capaz de emo- } \\
\text { cionar, debe tratar temas de interés } \\
\text { del lector, debe motivar y provocar } \\
\text { cambios en el lector, debe provocar } \\
\text { placer y entusiasmo en el lector debe } \\
\text { invitar a la lectura. }\end{array}$ \\
\hline
\end{tabular}

\section{Método}

Para llevar a cabo este estudio nos hemos situado en el contexto de estudiantes universitarios de primer año. En primer lugar, nos interesa identificar las teorías implícitas sobre comprensión de textos que predominan en estudiantes universitarios al ingreso a la universidad. En segundo lugar, nos interesa indagar en las teorías predominantes según grupo de formación, de manera de abordar los ámbitos del conocimiento y las teorías implícitas predominantes en las diversas disciplinas propias del contexto académico universitario. Dados estos objetivos, se realizará un estudio exploratorio descriptivo orientado a caracterizar a los estudiantes a partir de las teorías implícitas, determinando la presencia de estas teorías en los estudiantes de primer año. 


\subsection{Participantes}

Población. El universo de estudiantes matriculados vía PSU de primer año (año 2012) corresponde a 386 alumnos. Estos estudiantes corresponden mayoritariamente a alumnos egresados de colegios municipalizados y particulares subvencionados (75\%) mayoritariamente de la región de Magallanes, Chile.

Muestra. La muestra de estudio está constituida por 264 estudiantes de primer curso universitario que participaron voluntariamente en la investigación. El tamaño muestral es representativo de la población al nivel de confianza del $95 \%$. Los estudiantes proceden de las siguientes facultades: Facultad de Humanidades, Ciencias Sociales y de la Salud, Facultad de Ciencias Económicas, Administrativas y Jurídicas, Facultad de Ciencias y Facultad de Ingeniería. Dado que uno de los objetivos del estudio es determinar diferencias de acuerdo al ámbito de formación del estudiante, se ha reagrupado a los estudiantes en relación a su disciplina. La tabla siguiente presenta a los estudiantes participantes organizados en los cuatro grupos de formación empleados en los análisis.

Tabla 2. Número de estudiantes participantes distribuidos por grupo de formación.

\begin{tabular}{|l|l|l|}
\hline & Grupos de formación & $\mathbf{N}$ \\
\hline Educación & $\begin{array}{l}\text { Ciencias Sociales y Educación: Psicología-Trabajo Social- Peda- } \\
\text { gogía Inglés- Básica- Castellano }\end{array}$ & 88 \\
\hline Salud & $\begin{array}{l}\text { Ciencias de la Salud: Enfermería-Terapia Ocupacional-Kinesi- } \\
\text { ología }\end{array}$ & 91 \\
\hline Economía & $\begin{array}{l}\text { Ciencias Económicas, Administrativas y Jurídicas: Derecho- In- } \\
\text { geniería Comercial }\end{array}$ & 56 \\
\hline Ingeniería & Ciencias de la Ingeniería: Ingeniería Plan Común & 29 \\
\hline & Total & 264 \\
\hline
\end{tabular}

\subsection{Instrumento}

Se elaboró un cuestionario para medir las teorías implícitas de comprensión lectora en los estudiantes universitarios. El cuestionario está constituido por 27 ítems, 9 por cada Teoría (Lineal, Interactiva y Literaria). Los ítems fueron elaborados incluyendo contenidos teóricos de los tres conceptos centrales en la comprensión de textos (comprensión, lector y texto).

La sub-escala caracterizada como Teoría Lineal está compuesta por 9 enunciados que implican una concepción de la comprensión vinculada a la decodificación de signos gráficos, extracción de información relevante, reconocimiento léxico, interpretación de sentidos y reproducción literal del texto. Por ejemplo: "Considero que la comprensión solo es posible si se interpreta la totalidad de las ideas que el autor plantea". La sub-escala caracterizada como Teoría Interactiva está compuesta 
por 9 enunciados que implican una concepción de la comprensión que supera el procesamiento lineal, en el cual el lector debe interpretar los significados integrando sus conocimientos previos. Por ejemplo: "Creo que los lectores hábiles confían menos en el factor visual y recurren principalmente a los conocimientos que poseen acerca de lo que leen". La sub-escala caracterizada como Teoría Literaria está compuesta por 9 enunciados que implica concebir la comprensión basados en la valoración estética de un texto, en la identificación de personajes, temas y problemas humanos. Por ejemplo: "Comprender es imaginar, disfrutar, valorar estéticamente un texto, para que haya comprensión debe existir empatía entre el lector y los personajes".

Para responder el cuestionario, se solicitó a los estudiantes que informaran sobre su grado de acuerdo con la información contenida en cada ítem en una escala tipo Likert de 5 puntos (1-nada, 2-poco, 3-algo, 4-bastante, 5-mucho). El cuestionario de teorías implícitas ha alcanzado buenos índices de fiabilidad en todas las sub-escalas: $\alpha=.61$ para la Teoría Lineal, $\alpha=.71$ para la Teoría Interactiva y $\alpha=.73$ para la Teoría Literaria.

\subsection{Procedimiento}

Para acceder a la muestra se solicitó a los Jefes de Carrera autorización para aplicar el cuestionario al inicio del año académico (marzo, 2012). A los estudiantes se les informó acerca del estudio y se les entregó una carta de Consentimiento Informado que debieran firmar en caso de acceder a participar del estudio. En ella se especificaban los siguientes datos: objetivos del estudio, duración y lugar de aplicación de instrumentos, derecho de los participantes de conocer los resultados finales del estudio y el procedimiento que se ocupará para ello, procedimiento y responsable del resguardo y almacenamiento de la información recolectada, explicitación de fines asociados a la investigación y utilización de datos, explicitación de derecho a retiro y al rechazo de la participación sin consecuencias de ningún tipo y la correspondiente formulación de preguntas y respuestas de los sujetos.

\section{Resultados}

Los resultados generales indican que las tres teorías implícitas presentan correlaciones significativas entre ellas. Entre la Teoría Lineal y la Teoría Interactiva se alcanza una correlación de $.636(\mathrm{p}<.000)$, con la Teoría Literaria la correlación es de .474 ( $\mathrm{p}<.000)$ y entre la Teoría Interactiva y la Teoría Literaria la correlación es de .611 ( $\mathrm{p}<.000)$. En todos los grupos de estudio predomina la aceptación de la Teoría Lineal, alcanzando los valores más elevados en las medias ponderadas de las sub-escalas (Tabla 3). 
Tabla 3. Medias y desviaciones típicas teorías implícitas según el grupo de estudio.

\begin{tabular}{|l|l|l|l|l|l|l|}
\hline & \multicolumn{2}{|c|}{ Teoría Lineal } & \multicolumn{2}{c|}{ Teoría Interactiva } & \multicolumn{2}{c|}{ Teoría Literaria } \\
\hline & $M$ & $S_{\chi}$ & $M$ & $S_{\chi}$ & $M$ & $S_{\chi}$ \\
\hline Educación & 2.94 & 0.41 & 2.87 & 0.42 & 2.85 & 0.41 \\
\hline Salud & 3.08 & 0.39 & 3.04 & 0.38 & 2.96 & 0.41 \\
\hline Economía & 2.91 & 0.62 & 2.76 & 0.62 & 2.61 & 0.66 \\
\hline Ingeniería & 3.04 & 0.40 & 2.85 & 0.40 & 2.74 & 0.60 \\
\hline
\end{tabular}

Como puede apreciarse en los diagramas de caja (Gráfico 1), los cuatro grupos de estudio se distribuyen homogéneamente en la sub-escala de la Teoría Lineal, encontrándose las mayores diferencias, básicamente, en la sub-escala de la Teoría Literaria: 

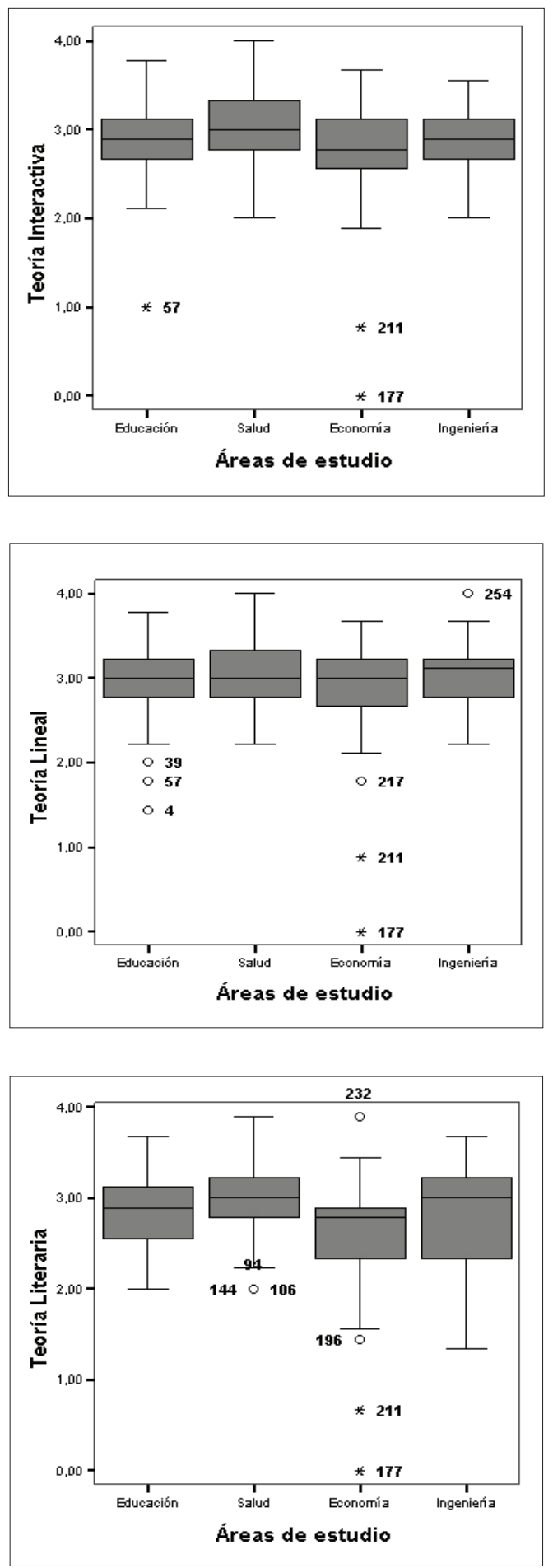

Gráfico 1. Diagramas de caja de las sub-escalas de teorías implícitas de comprensión. 
Los análisis multivariantes indican la influencia del grupo de estudio, $\mathrm{F}=2.77$, $\mathrm{p}<.005$ y de la intersección, $\mathrm{F}=3816.47, \mathrm{p}<.000$. El modelo corregido obtiene un $\mathrm{R}^{2}=.065$. En la sub-escala de la Teoría Lineal las diferencias no llegan a alcanzar significación estadística (Tabla 4).

Tabla 4. Contrastes de medias y desviaciones típicas según los grupos de estudio.

\begin{tabular}{|c|c|c|c|c|c|}
\hline & $\mathbf{M}$ & Sx & $\mathbf{F}$ & $p$ & Eta \\
\hline \multicolumn{6}{|c|}{ Teoría Lineal } \\
\hline Educación & 2.94 & 0.41 & \multirow{4}{*}{2.27} & \multirow{4}{*}{.081} & \multirow{4}{*}{.161} \\
\hline Salud & 3.08 & 0.39 & & & \\
\hline Economía & 2.91 & 0.62 & & & \\
\hline Ingeniería & 3.04 & 0.40 & & & \\
\hline \multicolumn{6}{|c|}{ Teoría Interactiva } \\
\hline Educación & 2.87 & 0.42 & \multirow{4}{*}{4.78} & \multirow{4}{*}{.003} & \multirow{4}{*}{.230} \\
\hline Salud & 3.04 & 0.39 & & & \\
\hline Economía & 2.76 & 0.62 & & & \\
\hline Ingeniería & 2.85 & 0.40 & & & \\
\hline \multicolumn{6}{|c|}{ Teoría Literaria } \\
\hline Educación & 2.86 & 0.41 & \multirow{4}{*}{5.97} & \multirow{4}{*}{.001} & \multirow{4}{*}{.255} \\
\hline Salud & 2.95 & 0.41 & & & \\
\hline Economía & 2.61 & 0.66 & & & \\
\hline Ingeniería & 2.74 & 0.60 & & & \\
\hline
\end{tabular}

Para conocer cómo se distribuyen los estudiantes atendiendo a las teorías implícitas de comprensión según los grupos de estudio, hemos segmentado la muestra en tres niveles para cada una de las teorías implícitas de comprensión: bajo, medio y alto. Para la segmentación hemos calculado los valores en la muestra total realizando tres grupos iguales porcentualmente. No existen diferencias en la distribución de la Teoría Lineal, $\chi 2=4.93 . \mathrm{p}=.552$, el $70 \%$ de los estudiantes de todos los grupos de estudio se ubican en los niveles medio y alto, predominan en el nivel alto los estudiantes de Ingeniería, 51,7\%. Tampoco se producen diferencias significativas en la distribución de la Teoría Interactiva, $\chi 2=10.43 . \mathrm{p}=.108$. Sin embargo, en la Teoría Literaria sí aparecen diferencias, $\chi 2=26.55, \mathrm{p}<.000$, en la dirección de mayores porcentajes del nivel bajo en los estudiantes de Economía e Ingeniería; el 73\% de los estudiantes de Educación se ubican entre los niveles medio y alto. Solamente el 19\% de los estudiantes de Salud se sitúan en el nivel bajo.

\section{Discusión y conclusiones}

En términos generales es posible señalar la presencia de las tres teorías implícitas sobre comprensión entre estudiantes universitarios de primer año: Teoría Lineal, Interactiva y Literaria. Esta clasificación resulta relevante de acuerdo a diversos autores (Schraw, 2000; Vélez, 2006; Hernández, 2008; Moore \& Narciso, 2011) por cuanto 
constituye un referente a considerar entre los diversos factores que contribuyen a desarrollar un conocimiento mayor del lector (Winneburg, 1991; Pressley \& Afferbach; 1995; Bahloul, 2002).

Un aspecto que se destaca en este estudio es el hecho de que la Teoría Lineal predomina en todos los grupos de formación, siendo la que obtiene los valores medios más elevados en todos los grupos. Esta tendencia también puede observarse en la distribución porcentual de los estudiantes en esta teoría, pues todos los grupos de formación se ubican en los niveles medio y alto. No obstante ello, los estudiantes de Ingeniería predominan en el nivel alto. Este resultado podría estar relacionado con la mayor necesidad de preservar aspectos textuales relacionados con la información de textos instrumentales y de aprendizaje en este grupo formativo.

Por su parte, la Teoría Interactiva no presenta diferencias significativas en la distribución de los cuatro grupos de estudiantes. En cambio, esta distribución sí presenta diferencias en la Teoría Literaria, por cuanto el mayor porcentaje de estudiantes de los grupos de Economía e Ingeniería se ubica en el nivel bajo. Asimismo, este resultado se observa en que la Teoría Literaria obtiene la media más baja en el grupo de Ciencias Económicas, Administrativas y Jurídicas. En esta teoría, un elevado porcentaje de estudiantes de Educación se ubica entre los niveles medio y alto. Posiblemente vinculado con la mayor relevancia de la lectura de textos literarios en la formación y posterior desarrollo profesional de estas especialidades.

La presencia de la Teoría Lineal y la Teoría Interactiva en los estudiantes universitarios, puede complementarse con los hallazgos realizados en el marco de estudios metacognitivos de Peronard, Velázquez, Crespo y Viramonte (2002) y Peronard (2005), en tanto dichos estudios observan que los estudiantes de enseñanza básica evolucionan desde una concepción lineal hacia una concepción interactiva en la medida que avanzan en el proceso formativo, información que queda reflejada en la correlación existente entre las tres teorías en nuestros estudiantes. Nuestro estudio podría proyectarse a identificar esta evolución en estudiantes universitarios.

Como podemos observar, la distribución de los resultados varía en función de los grupos de formación. Se agrupan, por una parte, Educación y Salud, con un desarrollo similar de las tres teorías de comprensión; por otra parte, se agrupan Economía e Ingeniería, con un claro predominio de la Teoría Lineal. La Teoría Lineal, como hemos señalado destaca la centralidad del texto, la búsqueda de información, en otras palabras, la necesidad de asegurar una lectura fiel a los contenidos transmitidos por el texto y el autor. Lo anterior nos plantea la necesidad de realizar estudios más amplios con estudiantes de diferentes años en las distintas carreras para analizar la influencia de la formación en el desarrollo de las teorías implícitas de comprensión. Sería interesante poder realizar estudios longitudinales de seguimiento de estudiantes durante sus estudios para conocer la evolución como comunidad discursiva específica como parte identitaria de su formación. 
El valor medio elevado de la Teoría Lineal en todos los grupos de estudio nos indica que en el contexto universitario, y frente a demandas académicas, los estudiantes activan la Teoría Lineal, lo que puede interpretarse como la necesidad de una lectura con fines de aprendizaje, para la adquisición de conocimientos. Igualmente, podría reflejar su actividad cotidiana de lectura, básicamente de textos expositivos, que requieren de la Teoría Lineal para su comprensión y posterior evaluación de contenidos.

La identificación de estas teorías constituye una aproximación inicial a reconocer aquellos modelos que en el ámbito de la comprensión se han internalizado en los estudiantes esta fase constituye un primer intento por iniciar procesos de redescripción (Pozo et al., 2006) con la finalidad de identificar elementos contextuales y personales que constituyen las teorías implícitas de los lectores.

Los resultados tienen además una clara connotación aplicada en el sentido de que la formación de los profesionales que tienen entre sus competencias el desarrollo de los hábitos lectores (campo educativo) precisarían el desarrollo de la Teoría Literaria, como una forma de que la actividad lectora promueva progresivamente una Teoría Interactiva que implica mayores exigencias y que permita superar los problemas asociados al rendimiento lector. Estos resultados deben interpretarse con cautela debido al tamaño reducido de la muestra, ya que los sujetos proceden de una única universidad y, fundamentalmente, al carácter transversal de los datos, lo cual, no nos permite establecer relaciones de tipo causal.

En síntesis, podemos señalar que las teorías implícitas sobre la comprensión están presentes en el grupo total de estudiantes de manera homogénea; sin embargo, esta homogeneidad no es tan evidente cuando se comparan los grupos de formación. Lo anterior nos plantea la necesidad de realizar estudios más amplios con estudiantes de diferentes años en las distintas carreras para analizar en profundidad la influencia de la formación en el desarrollo de las teorías implícitas de comprensión. Sería interesante poder realizar estudios longitudinales de seguimiento de estudiantes durante sus estudios para conocer la evolución como comunidad discursiva específica como parte identitaria de su formación.

Finalmente, la presencia de la Teoría Lineal y de la Teoría Interactiva en los estudiantes universitarios puede complementarse con los hallazgos realizados en el marco de estudios metacognitivos de Peronard et al. (2002) y Peronard (2005), en tanto dichos estudios observan que los estudiantes de enseñanza básica evolucionan desde una concepción lineal hacia una concepción interactiva en la medida que avanzan en el proceso formativo, información que queda reflejada en la correlación existente entre las tres teorías en nuestros estudiantes. Nuestro estudio podría proyectarse a identificar esta evolución en estudiantes universitarios. 


\section{REFERENCIAS BIBLIOGRÁFICAS}

Bahloul, J. (2002). Lecturas precarias; estudio sociológico sobre los poco lectores. México: Fondo de Cultura Económica.

Carlino, P. (2003). Leer textos cientificos y académicos en la educación superior: Obstáculos $y$ bienvenidas a una cultura nueva. Ponencia presentada en el $6^{\circ}$ Congreso Internacional de la Promoción de la Lectura y el Libro, Buenos Aires, Argentina.

Castro, A. (2006). Teorías implícitas del liderazgo, contexto y capacidad de conducción. Anales de Psicología, 22(1), 89-97.

Catalán, J. (2010). Teorías subjetivas. Aspectos teóricos y prácticos. La Serena: Editorial Universidad de la Serena.

Contreras, S. (2010). Las creencias curriculares de los profesores de ciencias: Una aproximación a las teorías implícitas sobre el aprendizaje. Horizontes Educacionales, 15(1), 23-36.

Colomer, T. \& Camps, A. (1996). Enseñar a leer, enseñar a comprender. Madrid: Celeste Ediciones.

Dann, H. (1990). Subjective theories: A new approach to Psychological research and Educational practice. En G. Semin \& K. Gergen (Eds.), Everyday Understanding (pp. 227-243). Londres: Sage Publications.

De Vega, M. (1998). La psicología cognitiva: Ensayo sobre un paradigma en transformación. Anuario de Psicología, 1, 20-44.

De Vega, M. (2004). Potenciales evocados cerebrales asociados al efecto de imaginibilidad en el procesamiento semántico. Revista de Neurología, 39(12), 1123-1128.

Dubois, M. (1987). El proceso de Lectura. De la Teoría a la Práctica. Buenos Aires: Aique.

Estrada, C., Yzerbit, V. \& Serón, E. (2004). El efecto del esencialismo psicológico sobre las teorías ingenuas de las diferencias grupales. Psicothema, 16(2), 181186.

Estrada, C., Oyarzún, M. \& Yzerbit, V. (2007). Teorías implícitas y esencialismo psicológico: Herramientas conceptuales para el estudio de la relaciones entre y dentro de los grupos. Psikhe, 16(1), 111-121.

Eyzaguirre, B. \& Fontaine, L. (2008). Las escuelas que tenemos. Santiago: Centro de Estudios Públicos. 
García-Jiménez, E. (1988). Las teorías implícitas sobre evaluación en el proceso de pensamiento de los profesores. En L. Villar (Coord.), Conocimiento, creenciasy teorias de los profesores (pp. 97-120). Alicante: Marfil.

Gómez, V. \& Guerra, P. (2012). Teorías implícitas respecto a la enseñanza y el aprendizaje: ¿Existen diferencias entre profesores en ejercicio y estudiantes de pedagogía? Estudios Pedagógicos, 38(1), 25-43.

Goodman, Y. \& Goodman, K. (1994). To err is human: Learning about language processes by analyzing miscues. En H. Singer \& B. Rudell (Eds.), Theoretical models and processes of reading (pp.104-123). Newark, Delaware: IR A \& Erlbaum.

Gough, P. (1994). One second of reading. En H. Singer \& B. Rudell (Eds.), Theoretical models and processes of reading (pp. 661-686). Newark, Delaware: IR A \& Erlbaum.

Groeben, N. (1990). Subjective theories and the explanation of human action. En G. R. Semin \& K. Gergen (Eds.), Every day understanding. Social and scientific implications (pp. 19-44). Londres: Sage.

Hernández, G. (2008). Teorías implícitas de lectura y conocimiento metatextual. Revista Mejicana de Investigación Educativa, 13(38), 737-771.

Ibáñez, R. (2008). Comprensión de textos académicos escritos en inglés: Relación entre nivel de logro y variables involucradas. Revista Signos. Estudios de Lingüistica, 41(67), 203-229.

Jamet, E. (2006). Lectura y éxito escolar. Buenos Aires: Fondo de Cultura Económica.

Jiménez, A. (2002). Nivel educativo y teorías implicitas sobre la enseñanza: Estructura de creencias en los docentes de educación infantil y primaria, secundaria y superior. Tesis doctoral. Pontificia Universidad Católica de Santiago, Chile.

Karmiloff-Smith, A. (1997). Auto-organización y cambio cognitivo. Substratum, 1(1), 19-43.

Karmiloff-Smith, A. \& Inhelder, B. (1984). If you want to get ahead, get a theory. Cognition, 3(3), 195-212.

Kintsch, W. (2002). On the notions of theme and topic in psychological process models of text comprehension. En M. Louwerse \& W. van Peer (Eds.), Thematics: Interdisciplinary studies (pp. 151-170). Ámsterdam: John Benjamins.

Kintsch, W. (2008). Symbol systems and perceptual representations. En M. De Vega, A. Glenberg \& A. Graesser (Eds.), Symbols and embodiment (pp. 145-164). Oxford: Oxford University Press. 
Laberge, D. \& Samuels, S. J. (1994). Toward a theory of automatic information processing of reading. En H. Singer \& B. Rudell (Eds.) Theoretical models and processes of reading (pp. 689-718). Newark, Delaware: IRA \& Erlbaum.

Linuesa, M. \& Domínguez, A. (1999). La enseñanza de la lectura. Enfoque psicolingüístico y sociocultural. Madrid: Pirámide.

Makuc, M. (2008) Teorías implícitas de los profesores acerca de la comprensión de textos. Revista Signos. Estudios de Lingüistica, 41(68), 403-422.

Makuc, M. (2011). Teorías implícitas sobre comprensión textual y la competencia lectora de estudiantes de primer año de la Universidad de Magallanes. Estudios Pedagógicos, 37(1), 236-253.

Makuc, M. (2012). La comprensión textual en el discurso de estudiantes universitarios: Aproximación desde el significado ideacional manifestado en la cláusula. CODIS, Working Papers, 1, 23-40.

Makuc, M. (2013). Teorias implícitas de los profesores acerca de la comprensión de textos. Punta Arenas: Universidad de Magallanes.

Marinkovich, J., Peronard, M. \& Parodi, G. (2006). Lectes. Programa de lectura y escritura para $7^{\circ}$ y $8^{\circ}$ básicos. Guía metodológica y orientaciones teóricas. Valparaíso: Ediciones Universitarias de Valparaíso.

Marrero, J. (1988). Las teorias implíitas y la planificación del profesor. Tesis doctoral no publicada. Universidad de la Laguna. España.

Marrero, J. (2009). Escenarios, saberes y teorías implícitas del profesorado. En J. Marrero (Ed.), El pensamiento reencontrado (pp. 8-44). Barcelona: Octaedro.

Martínez, J. (2009). Las teorías implícitas como conocimiento práctico del profesorado en formación inicial. En J. Marrero (Ed.), El pensamiento reencontrado (pp. 208217). Barcelona: Octaedro.

McKoon, G. \& Ratcliff, R. (1998). Memory-based languaje processing: Psycholinguistic research in the 1990s. Annual Review, 49, 25-42.

Mitchell, J. (1995). Teacher's implicit theories concerning questioning. British Educational Research Journal, 1, 69-84.

Moore, P. \& Narciso, E. (2011). Modelos epistémicos de la lectura en estudiantes mexicanos. Revista Mexicana de Investigación Educativa, 16(51), 1197-1225.

OCDE (2010). Resultados PIS A 2009: Resumen ejecutivo [en línea]. Disponible en: http:// www.oei.es 
Paris, S., Lipson, M. \& Wixson, K. (1994). Becoming a strategic reader. En H. Singer \& B. Rudell (Eds.), Theoretical models and processes of reading (pp. 778-862). Newark, Delaware: IRA \& Erlbaum.

Parodi, G. (1999). Relaciones entre lectura y escritura: Una perspectiva cognitiva discursiva. Valparaíso: Ediciones Universitarias de Valparaíso.

Parodi, G. (2005). La comprensión del discurso especializado escrito en ámbitos técnico-profesionales: ¿Aprendiendo a partir del texto? Revista Signos. Estudios de Lingüistica, 38(58), 221-267.

Parodi, G. (2007). Comprensión y aprendizaje a partir del discurso especializado escrito: Teoría y empiria. En G. Parodi (Ed.), Lingüistica de corpus y discursos especializados: Puntos de mira (pp. 223-258). Valparaíso: Ediciones Universitarias de Valparaíso.

Parodi, G. (2009). El corpus académico y profesional del español PUCV 2006: Semejanzas y diferencias entre los géneros académicos y profesionales. Estudios Filológicos, 44, 123-147.

Pearson, D. \& Stephens, D. (1994). Learning about literacy: A 30-year journey. En H. Singer \& B. Rudell (Eds.), Theoretical Models and processes of reading (pp. 22-103). Newark, Delaware: IRA \& Erlbaum.

Peredo, M. (2003). La identidad lectora y los pensamientos que se activan durante la lectura de los textos de auto ayuda. Ponencia presentada en el $6^{\circ}$ Congreso Internacional de la Promoción de la Lectura y el Libro, Buenos Aires, Argentina.

Peronard, M. (2005). La metacognición como herramienta didáctica. Revista Signos. Estudios de Lingüística, 38(57), 61-74.

Peronard, M., Velásquez, M., Crespo, N. \& Viramonte, M. (2002). Conocimiento metacognitivo del lenguaje escrito: Instrumento de medida y fundamentación teórica. Infancia y Aprendizaje, 25(2), 131-145.

Pozo, J. (2001). Humana mente. El mundo, la conciencia y la carne. Madrid: Morata.

Pozo, J. (2003). Adquisición del conocimiento. Madrid: Morata.

Pozo, J. \& Carretero, M. (1987). Del pensamiento formal a las concepciones espontáneas: ¿Qué cambia en la enseñanza de la ciencia? Infancia y Aprendizaje, $38,35-42$.

Pozo, J., Pérez, M. P., Sanz, A. \& Limón, M. (1992). Las ideas de los alumnos sobre la ciencia como teorías implícitas. Infancia y Aprendizaje, 57, 3-21. 
Pozo, J. \& Rodrigo, M. (2001). Del cambio de contenido al cambio representacional en el conocimiento conceptual. Infancia y Aprendiraje, 24, 407-423.

Pozo, J., Scheuer, N., Mateos, M. \& Pérez-Echeverría, M. (2006). Las teorías implícitas sobre el aprendizaje y la enseñanza. En J. Pozo, N. Scheuer, M. Pérez-Echeverría, M. Mateos, E. Martín \& M. de la Cruz (Eds.), Nuevas formas de pensar la enseñanza y el aprendizaje. Las concepciones de profesores y alumnos (pp. 95127). Barcelona: Grao.

Pressley, M. \& Afferbach, P. (1995). Verbal prococols of reading: The nature of constructively responsive reading. Hillsdale, N. J.: Lawrence Erlbaum.

Rodrigo, M. J. (1985). Las teorías implícitas en el conocimiento social. Infancia y Aprendizaje, 31-32, 145-156.

Rodrigo, M. J. (1993). Representaciones y procesos en las teorías implícitas. En M. J. Rodrigo, M. J. Rodríguez \& J. Marrero (Eds.), Las teorías implíitas. Una aproximación al conocimiento cotidiano (pp. 95-117). Madrid: Visor.

Rodrigo, M. J. (1994a). El hombre de la calle, el científico y el alumno: ¿Un solo constructivismo o tres? Investigación en la Escuela, 23, 7-16.

Rodrigo, M. J. (1994b). Etapas, contextos, dominios y teorías implícitas en el conocimiento social. En M. J. Rodrigo (Ed.), Contexto y desarrollo social (pp. 26-46). Madrid: Síntesis.

Rodrigo, M. J. (1997). Del escenario sociocultural al constructivismo episódico: Un viaje al conocimiento escolar de la mano de las teorías implícitas. En M. J. Rodrigo \& J. Arnay (Comps.), La construcción del conocimiento escolar (pp. 177191). Barcelona: Paidós.

Rodrigo, M. J., Rodríguez, M. J. \& Marrero, J. (1993). Las teorías implícitas. Una aproximación al conocimiento cotidiano. Madrid: Visor.

Rodrigo, M. J. \& Correa, N. (2001). Representación y procesos cognitivos: Esquemas y modelos mentales. En C. Coll, J. Palacios \& A. Marchesi (Comps.), Desarrollo psicológico y educación. Psicología de la educación escolar (pp. 117-135). Madrid: Alianza.

Rodríguez, A. (1993). El conflicto intergrupal desde las teorías implícitas. En M. J. Rodrigo \& J. Arnay (Comps.), La construcción del conocimiento escolar (pp. 177191). Barcelona: Paidós.

Rodríguez, E. (2001). Teoría implícita y formación inicial del profesorado de Educación Media. Revista Enfoques Educacionales, 3(2), 145-155.

Ruiz, M. (2009). Evaluación de lengua escrita y dependencia de lo literal. Barcelona: Graó. 
Rumelhart, D. (1997). Hacia una comprensión de la comprensión. En E. Rodríguez \& E. Laguer (Comps.), La lectura (pp. 25-51). Santiago de Cali: Editorial Universidad del Valle.

Schraw, G. (2000). Reader beliefs and meaning construction in narrative text. Journal of Educational Psychology, 92(1), 96-106.

Schraw, G. \& Bruning, R. (1996). Readers' implicit models of reading. Reading Research Quarterly, 31(3), 290-305.

Scheuer, N. \& Pozo, J. (2006). ¿Qué cambia en las teorías implícitas sobre el aprendizaje y la enseñanza? Dimensiones y procesos de cambio. En J. Pozo, N. Scheuer, M. Pérez-Echeverría, M. Mateos, E. Martín \& M. de la Cruz (Eds.), Nuevas formas de pensar la enseñanza y el aprendizaje. Las concepciones de profesores y alumnos (pp. 375-398). Barcelona: Grao.

Triana, B. (1991). Las concepciones de los padres sobre el desarrollo: Teorías personales o teorías culturales. Infancia y Aprendizaje, 54, 19-39.

Triana, B. \& Rodrigo, M. J. (1985). El concepto de infancia en nuestra sociedad: Una investigación sobre teorías implícitas de los padres. Infancia y Aprendiraje, 3132, 157-171.

van Dijk, T. (2001). Algunos principios de la teoría del contexto. Revista Latinoamericana de Estudios del Discurso, 1(1), 69-82.

van Dijk, T. \& Kintsch, W. (1983). Strategic of discourse comprehension. Nueva York: Academic Press.

Velásquez, M., Cornejo, C. \& Roco, A. (2008). Evaluación de la competencia lectora en estudiantes de primer año de carreras del Área Humanista y carreras del Área de la Salud en tres universidades del Consejo de Rectores. Estudios Pedagógicos, 33(1), 123-138.

Vélez, G. (2006). Las autobiografías lectoras como autobiografía de aprendizaje. En J. Pozo, N. Scheuer, M. Pérez-Echeverría, M. Mateos, E. Martín \& M. de la Cruz (Eds.), Nuevas formas de pensar la enseñanza y el aprendizaje. Las concepciones de profesores y alumnos (pp. 307-319). Barcelona: Grao.

Wineburg, S. (1991). On the reading of historical texts: Notes on the breach between school and academy. American Educational Research Journal, 28(3), 495-520. 


\section{NOTA}

1 Artículo elaborado en el marco del Proyecto FONDECYT REGULAR (№ 1120891): "Las Teorías implícitas sobre la comprensión y las estrategias del lector: aspectos de una relación dinámica y significativa en el desarrollo de las competencias lectoras de estudiantes universitarios de primer año". El presente artículo se ha realizado con la colaboración internacional de la investigadora Dra. María Elisa Larrañaga, Universidad de Castilla la Mancha, España. 